\title{
Enthüllung einer Erinnerungstafel zu Ehren von Wilhelm Killing und Karl Weierstraß in Braniewo'
}

Ulf Rehmann und Andrzej Szczepański

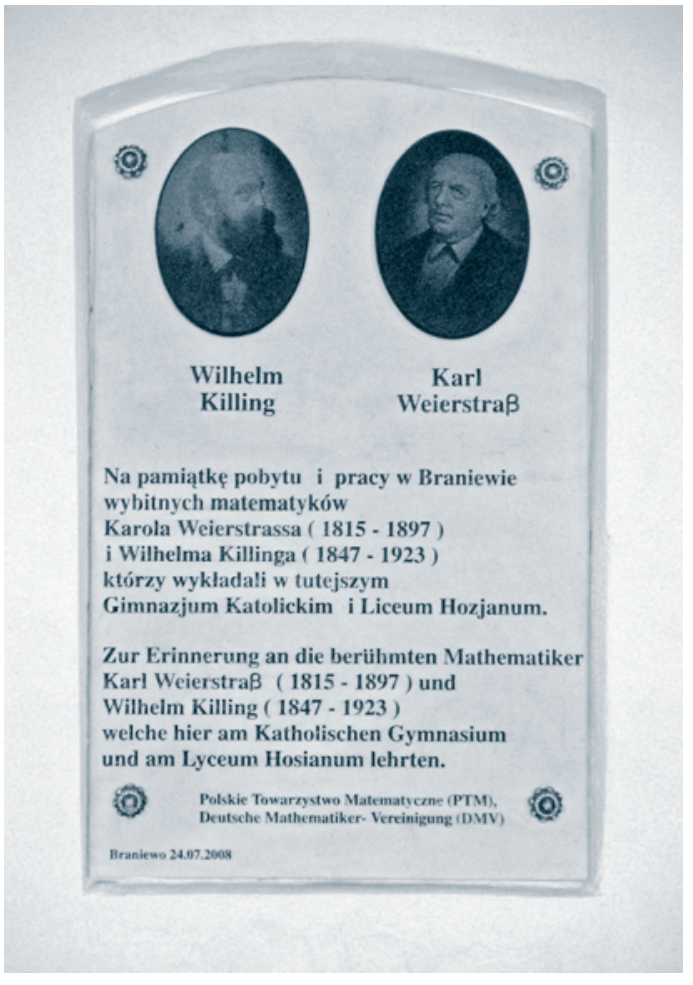

Gibt man in Google oder in MathSciNet oder im Zentralblatt für Mathematik den Suchbegriff „The Greatest Mathematical Paper of All Time" ein, so erhält man als Ergebnisse sowohl den Namen als auch das bedeutende Werk Wilhelm Killings (1847-1923). Insbesondere wird man verwiesen auf Killings Klassifikation der einfachen Lie-Algebren über den komplexen Zahlen, die er während seiner Zeit als Professor am Lyceum Hosianum in Braunsberg - oder Braniewo, wie die Stadt auf polnisch heißt - gefunden hat. $^{3}$

Killings Ergebnisse wurden 1888-1890 in vier Folgearbeiten in den Mathematischen Annalen [6] veröffentlicht. Der Inhalt dieser vier Arbeiten war Anlass für den Titel eines biografischen Artikels über Killing von A. J. Coleman (cf. [I]: "The Greatest Mathematical Paper of All Time”), der zum hundertjährigen Publikations-Jubiläum im Mathemetical Intelligencer erschien.

Colemans Bewunderung für das Werk Killings wurde denn auch klar von anderer kompetenter Seite geteilt: In Jean Dieudonnés Besprechung von Colemans Intelligencer-Artikel in den Mathematical Reviews heißt es: Killings result became a most important milestone in modern mathematics. Das lässt sich leicht durch einen Blick in die Geschichte der Mathematik im vergangenen Jahrhundert bestätigen: Immer wieder wurde Killings Resultat besprochen, umgearbeitet, vereinfacht und auf unterschiedliche Gebiete verallgemeinert, und dies von bedeutenden Mathematikern wie $E$. Cartan in seiner Thèse (I894), H. Weyl (1925), B. L. van der Waerden (1933), H. S. M. Coxeter (1934), E. Witt (194I), E. Stiefel (1942), E. D. Dynkin (1947), C. Chevalley (1955, 196I ff.), J. Tits (1966 ff.), V. G. Kac und R. V. Moody (1968), F. Bruhat ( 1972 ff.), um nur einige aufzuzählen.

Für Killings Einfluss auf unsere alltägliche mathematische Sprache lassen sich etliche Beispiele finden: Der Begriff "Charakteristische Gleichung" einer Matrix, heute jedem Anfänger-Studenten der Mathematik wohlbekannt, geht auf ihn zurück (cf. [6, II, p. 2]), und in verschiedenste mathematische Gebiete hat der Begriff „Halbeinfach“ Eingang gefunden - erfunden ebenfalls von Killing in [6, III, p. 74], wo er schreibt:

Solange ein besserer Name fehlt, möge es gestattet sein, eine solche (Gruppe) als eine halbeinfache zu bezeichnen.

Offenbar wurde nie eine bessere Bezeichnung gefunden.

Eine ausführliche Beschreibung der Geschichte von Killings Klassifikationsresultat zusammen mit bibliografischen Hinweisen findet man in [9].

Killing war ein Schüler von Karl Weierstraß in Berlin, wo er 1872 promovierte. Nach mehreren Jahren als Lehrer an verschiedenen Höheren Schulen und mit mehreren Publikationen zu geometrischen Fragen erhielt er 1882 einen Lehrstuhl für Mathematik am Lyceum Hosianum in Braunsberg. Detaillierte biografische Beschreibungen des Wirkens der beiden Mathematiker Weierstraß und Killing haben J. Elstrodt [4], bzw. F. Lorenz [8] gegeben, weitere Informationen findet man aber auch im Jahresbericht der DMV in den Würdigungen von K. Lampe, 1897, [7] und von F. Engel, 1930, [5].

Im November 1996 hielt der erstgenannte Autor dieses Berichtes einen Vortrag über "Linear algebraic groups and related structures" an der Universität Bielefeld, in dem er den Intelligencer-Artikel von Coleman über Killing und dessen Wirken in Braunsberg/Braniewo erwähnte. Der damals im Auditorium sitzende zweite Autor - 
von der Danziger Universität - war beeindruckt zu erfahren, dass Wilhelm Killing zehn Jahre (1882-1892) in Braunsberg, das etwa 110 km nordöstlich von Danzig gelegen ist, als Professor am Lyceum Hosianum gewirkt hatte.

Im Spätsommer 1998 organisierte das Institut für Mathematik der Universität Danzig in Braniewo einen Workshop mit dem Titel "The Second Days of Hyperbolic Geometry in memoriam of Wilhelm Killing". A. J. Coleman schrieb hierzu eine Grußadresse [2].

Als uns damals bewusst wurde, dass auch Karl Weierstraß von 1848 bis 1856 als Oberschullehrer in Braunsberg gearbeitet hatte, reifte die Idee heran, eine Gedenktafel zu Ehren von W. Killing und K. Weierstraß zu stiften. Die Verwirklichung dieser Idee brauchte zehn Jahre; teils, weil Geld beschafft werden musste, teils, weil die Genehmigung nicht so einfach zu bekommen war.

Die Feierlichkeiten zur Enthüllung der Gedenktafel am 24. und 25. Juli 2008 wurden vom Institut für Mathematik der Universität Danzig und dem Starost von Braniewo organisiert ${ }^{4}$. Auf der Tafel findet sich ein kurzer Text in polnischer und deutscher Sprache mit der Information über das Wirken von W. Killing und K. Weierstraß in Braniewo als Lehrer und mit den Signaturen der Polskie Towarzystwo Matematyczne (PTM, die polnische mathematische Gesellschaft) und der Deutschen MathematikerVereinigung (DMV). Dem Ehren-Komitee gehörten die Rektoren der Danziger Universität und der Universität Ermland-Masuren in Olsztyn (Allenstein) an.

Die Feierlichkeiten begannen mit einer Messe zum Gedenken an W. Killing und K. Weierstraß, zelebriert durch den örtlichen römisch-katholischen Bischof Jacek Jezierski. Sodann begaben sich die etwa 60 Teilnehmer zum Gebäude des früheren Lyceum Hosianum, ${ }^{5}$ um die Gedenktafel zu enthüllen.

Erster Sprecher war der Starost von Braniewo, Leszek Dziąg. Sodann wurden Grußadressen verlesen vom Rektor der Universität Münster (von F. Lorenz) und dem Oberbürgermeister der Stadt Münster (von J. Elstrodt), da W. Killing später Professor an der Universität Münster war und weil die Städte Braniewo und Münster durch eine Europäische Städtepartnerschaft verbunden sind. Schließlich enthüllten L. Dziąg und A. Szczepański die Tafel, die dann vom Bischof geweiht wurde.

Es sollte hier bemerkt werden, dass dies die einzige Tafel an diesem Ort ist, die "weltliche“ Wissenschaftler ehrt, neben einer Reihe von älteren Gedenktafeln ehrwürdiger geistlicher Würdenträger.

Im Anschluss an die Enthüllung fand ein Kolloquium statt, bei dem Vorträge zum Leben und Wirken Killings und Weierstraß' und zu ihrem mathematischen Wirken gehalten wurden. Am Abend des ersten Tages gab es ein Konzert, und anschließend fand man sich zu einer Party des Bürgermeisters von Braniewo zusammen.

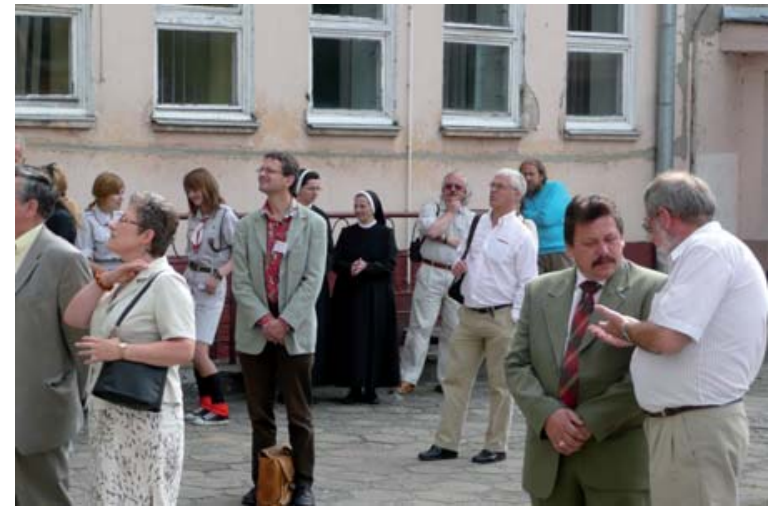

Teilnehmer der Zeremonie

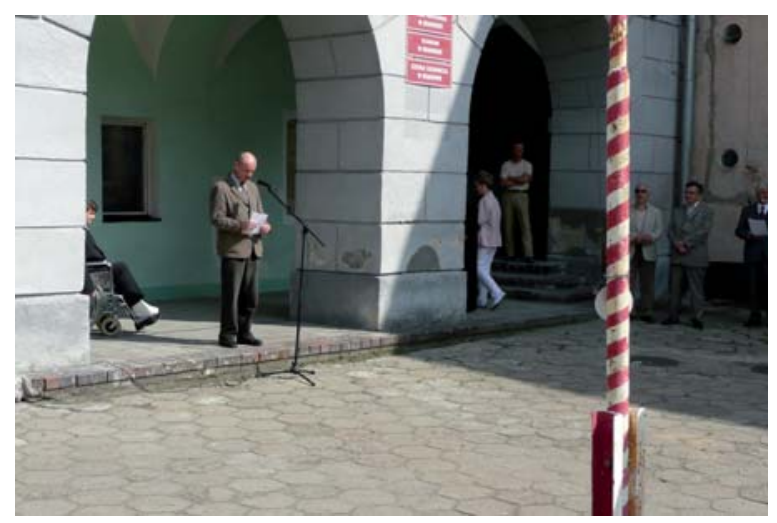

Die Zeremonie, geleitet durch Andrzej Szczepański

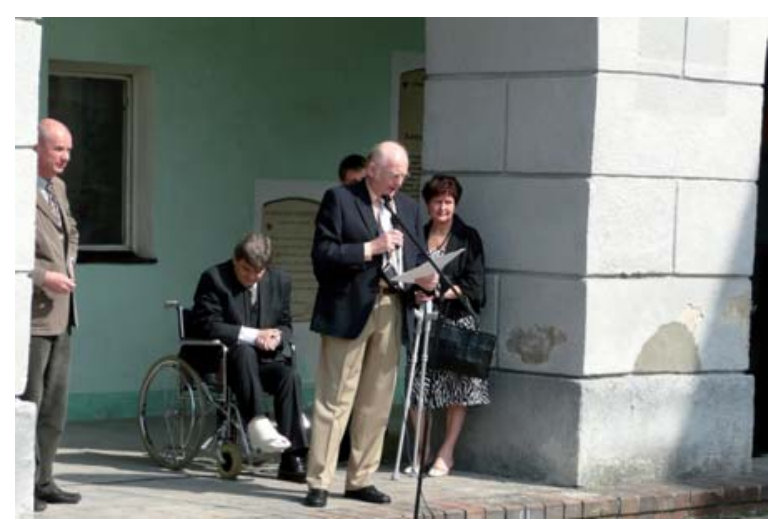

Jürgen Elstrodt verliest eine Grußbotschaft des Oberbürgermeisters der Stadt Münster, neben dem "Starost” von Braniewo

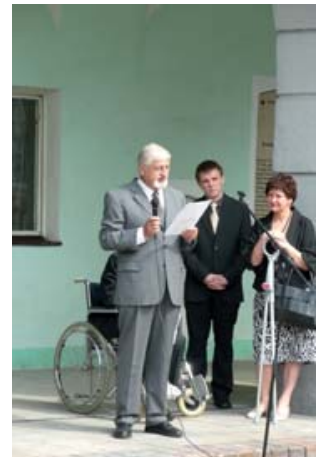

Falko Lorenz verliest die Grußbotschaft des des Rektors der Universität Münster 
Die Feierlichkeiten wurden ermöglicht durch finanzielle Unterstützung der Universitäten von Danzig und Münster, der Universität von Ermland-Masuren in Olsztyn, dem GWO Publishing House, dem Starost und der Stadt Braniewo sowie der Deutschen MathematikerVereinigung.

Weitere Informationen mit Photos und den Texten der historischen Vorträge von J. Elstrodt und F. Lorenz findet man in [II].

Vom 24. bis 26. März 2010 wird in Braniewo wieder ein gemeinsam organisiertes Kolloquium stattfinden: The Second W. Killing and K. Weierstraß Colloquium (http://mat.ug. edu.pl/kwwk/20l0/).

\section{Anmerkungen}

I. Die Original-Version dieses Artikels ist im Mathematical Intelligencer (0I/20I0) in der Rubrik "The Mathematical Tourist“ unter dem Titel „A Commemorative Plate for Wilhelm Killing and Karl Weierstraß" (DOI: 10.1007/s00283-009-9084-4) erschienen.

2. U. R.: Förderung durch den Sonderforschungsbereich 70I der DFG: Spektrale Strukturen und Topologische Methoden in der Mathematik, A. S.: Förderung durch den polnischen Grant $\mathrm{KBN}-0524 / \mathrm{H} 03 / 2006 / 3 \mathrm{I}$.

3. Im 19. Jahrhundert gehörte Braunsberg zu Ostpreußen, jetzt liegt diese Stadt in Polen.

4. Der Starost ist der Chef der Stadtverwaltung

5. Prof. Falko Lorenz vom Fachbereich Mathematik der Universität Münster wies uns freundlicherweise darauf hin, dass es sich tatsächlich um das frühere Katholische Gymnasium handelt, da das Gebäude des Lyceum Hosianum nicht mehr existiert.

\section{Literatur}

[I] A. J. Coleman: The Greatest Mathematical Paper of All Time. Mathematical Intelligencer, II (1989) no. 3, 39-38.

[2] A. J. Coleman: Killing in Braniewo, (in Polish). Wiadomości Matematyczne XXXV (1999), pp. I4I-144.

[3] MRI007036 (90f:01047) Review of [I] by Jean Dieudonné, Math. Reviews (1990).

[4] J. Elstrodt: Karl Weierstrass (1815-1897). http://math.univ. gda.pl/pdf/text_elstrodt.pdf

[5] F. Engel: Wilhelm Killing. Jahresbericht der Deutschen Mathematiker-Vereinigung 39, (1930), I40-154

[6] W. Killing: Die Zusammensetzung der stetigen endlichen Transformationsgruppen. I Math. Ann. 3I, (I888), 252-290 || ibid. 33, (1889), I-48 III ibid. 34, (1889), 57-I22 IV ibid. 36, (1890), $161-189$.

[7] K. Lampe: Karl Weierstraß. Jahresbericht der Deutschen Mathematiker-Vereinigung 6, 27-44

[8] F. Lorenz: Wilhelm Killing (1847-1923). http://math.univ.gda. pl/pdf/W.Killing-komplett.pdf

[9] Rehmann, Ulf: On Reflection Groups and semisimple Lie algebras, Remarks on the Article by E. Witt: Spiegelungsgruppen und Aufzählung halbeinfacher Liescher Ringe. In: Ernst Witt, Collected papers. Gesammelte Abhandlungen. Ed. by Ina Kersten. Springer-Verlag, Berlin, 1998, 247-255.

[10] http://math.univ.gda.pl/img/braniewo/slides/PI0I016I.html

[II] http://mat.ug.edu.pl/index.php?url=mod/article.php\&id=358

Andrzej Szczepański Institute of Mathematics, University of Gdańsk, ul. Wita Stwosza 57, 80-952 Gdańsk, Poland matas@paula.univ.gda.pl

Ulf Rehmann, Fakultät für Mathematik, Universität Bielefeld, Postfach I00I3I, 3350I Bielefeld. rehmann@math.uni-bielefeld.de 